\title{
Minimum-Energy Broadcast Routing in Static Ad Hoc Wireless Networks
}

\author{
P.-J. Wan* G. Călinescu* X.-Y. Li* O. Frieder*
}

\begin{abstract}
Energy conservation is a critical issue in ad hoc wireless networks for node and network life, as the nodes are powered by batteries only. One major approach for energy conservation is to route a communication session along the routes which requires the lowest total energy consumption. This optimization problem is referred to as minimum-energy routing. While minimum-energy unicast routing can be solved in polynomial time by shortest-path algorithms, it remains open whether minimum-energy broadcast routing can be solved in polynomial time, despite of the NP-hardness of its general graph version. Recently three greedy heuristics were proposed in [8]: MST (minimum spanning tree), SPT (shortest-path tree), and BIP (broadcasting incremental power). They have been evaluated through simulations in [8], but little is known about their analytical performance. The main contribution of this paper is the quantitative characterization of their performances in terms of approximation ratios. By exploring geometric structures of Euclidean MSTs, we have been able to prove that the approximation ratio of MST is between 6 and 12, and the approximation ratio of BIP is between $\frac{13}{3}$ and 12 . On the other hand, the approximation ratio of SPT is shown to be at least $\frac{n}{2}$, where $n$ is the number of receiving nodes. To our best knowledge, these are the first analytical results for minimum-energy broadcasting.
\end{abstract}

\section{INTRODUCTION}

Ad hoc wireless networks have received significant attention in recent years due to their potential applications in battlefield, emergency disaster relief and etc [7] [8]. Unlike wired networks or cellular networks, no wired backbone infrastructure is installed in ad hoc wireless networks. A communication session is achieved either through a single-hop transmission if the communication parties are close enough, or through relaying by intermediate nodes otherwise. Omnidirectional antennas are used by all nodes to transmit and receive signals. They are attractive in their broadcast nature. A single transmission by a node can be received by many nodes within its vicinity. This feature is extremely useful for multicasting/broadcasting communications. For the purpose of energy conservation, each node can dynamically adjust its transmitting power based on the distance of the receiving nodes and background noise. In the most common power-attenuation model [6], the signal power falls as $\frac{1}{r^{\kappa}}$ where $r$ is the distance from the transmitter antenna and $\kappa$ is the a constant between 2 and 4 dependent on the wireless environment. All receivers have the same power threshold for signal detection, which are typically normalized to one. With these assumptions, the power

* Department of Computer Science, Illinois Institute of Tech-
nology, Chicago, IL 60616. Emails: \{wan, calinescu, xli, nology, Chicago,
ophir\}@cs.iit.edu. required to support a link between two nodes separated by range $r$ is $r^{\kappa}$. A key observation is that relaying signal between nodes may results in lower transmission than communicating over large distances due to the nonlinear power attenuation. As a simple illustration, consider three nodes $\mathbf{p}_{\mathbf{1}}, \mathbf{p}_{\mathbf{2}}$ and $\mathbf{p}_{\mathbf{3}}$ with as $\left\|\mathbf{p}_{\mathbf{1}} \mathbf{p}_{\mathbf{2}}\right\|>\left\|\mathbf{p}_{\mathbf{1}} \mathbf{p}_{\mathbf{3}}\right\|$ in Figure 1 and assume $\kappa=2$. Node $\mathbf{p}_{\mathbf{1}}$ wants to send a message to node $\mathbf{p}_{\mathbf{2}}$. It has two options. It can transmit the signal directly to node $\mathbf{p}_{\mathbf{2}}$, with a energy consumption of $\left\|\mathbf{p}_{\mathbf{1}} \mathbf{p}_{\mathbf{2}}\right\|^{2}$. Alternatively, it can relay the message through node $\mathbf{p}_{\mathbf{3}}$ and have it retransmit to node $\mathbf{p}_{\mathbf{2}}$, with a total energy consumption of $\left\|\mathbf{p}_{\mathbf{1}} \mathbf{p}_{\mathbf{3}}\right\|^{2}+\left\|\mathbf{p}_{\mathbf{3}} \mathbf{p}_{\mathbf{2}}\right\|^{2}$. Therefore if the angle $\mathbf{p}_{\mathbf{1}} \mathbf{p}_{\mathbf{3}} \mathbf{p}_{\mathbf{2}}$ is obtuse, the second option consumes less total energy. A crucial issue is then how to find a routing with minimum total energy consumption for a given communication session. This problem is referred to as the Minimum-Energy Routing [7] [8].

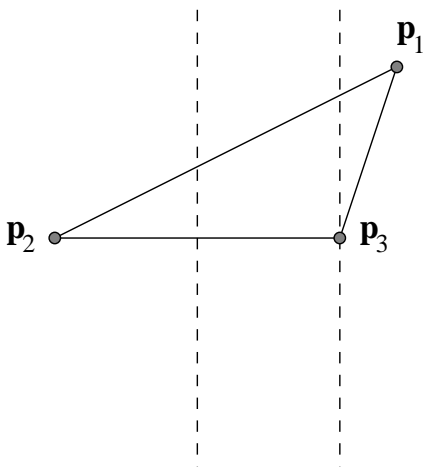

Fig. 1. Reduce energy consumption through relaying.

The minimum-energy broadcast/multicast routing in a simple ad hoc networking environment has been addressed by the pioneering work in [8]. To assess the complex trade-offs one at a time, the nodes in the network are assumed to be a point ${ }^{1}$ set randomly distributed in a twodimensional plane and there is no mobility. Nevertheless, as argued in [8], the impact of mobility can be incorporated into this static model because transmitter power can be adjusted to accommodate the new locations of the nodes, as necessary. In other words, the capability to adjust transmission power provides considerable "elasticity" to the topological connectivity, and hence may reduce the

${ }^{1}$ The terms node, point and vertex are interchangeable in this paper: node is a networking term, point is a geometric term, and vertex is a graph-theoretic term. 
need for hand-offs and tracking. In addition, as assumed in [8], there are sufficient bandwidth and transceiver resources. Under these assumptions, centralized (as opposed to distributed) algorithms were presented by [8] for minimum-energy broadcast/multicast routing. These centralized algorithms in this simple networking environment are expected to serve as the basis for further studies on the distributed algorithms in a more practical network environment with limited bandwidth and transceiver resources, as well as node mobility.

Three greedy heuristics were proposed in [8] for minimum-energy broadcast routing: MST (minimum spanning tree), SPT (shortest-path tree), and BIP (broadcasting incremental power). They have been evaluated through simulations in [8], but little is known about their analytical performances in terms of the approximation ratios. We believe that this analytical performance is very essential and more convincing in evaluating these heuristics. For the minimum-energy broadcast routing, one may come up with many seemingly reasonable greedy criteria. But it's hard to tell from simulation outputs which one is better or poorer. Indeed, all the three heuristics proposed in [8] only have subtle differences. For pure illustrative purpose, another a slight variation of BIP, which is referred to Broadcast Average Incremental Power (BAIP), will be introduced in Section III. These subtle differences, however, can have great impact on their performance ratios. In fact, we will show that the approximation ratios of MST and BIP are between 6 and 12 and between $\frac{13}{3}$ and 12 respectively; on the other hand, the approximation ratios of SPT and BAIP are at least $\frac{n}{2}$ and $\frac{4 n}{\ln n}-o(1)$ respectively, where $n$ is the number of nodes. To our best knowledge, these are the first quantiative characterizations of heuristics for minimum-energy broadcast routing.

The remaining of this paper is organized as follows. In Section II, we analyze the challenges for minimum-energy broadcast routing and briefly overview the three greedy heuristics developed in [8]. In Section III, we construct some bad instances to illustrate the poor performance of both SPT and BAIP. These instances lead to the lower bounds on the approximation ratios of SPT and BAIP. In Section IV, we construct instances to obtain lower bounds on the approximation ratios of MST and BIP. In Section $\mathrm{V}$, we derive upper bounds on the approximation ratios of MST and BIP. A cornerstone to the analysis of the upper bounds is an elegant structure property of Euclidean MST, which is explored in Section VI. Finally in Section VIIwe summarize our results and point out several future reseach problems.

\section{Preliminaries}

In this paper, we assume the network nodes are given as a finite point set $P$ in the two-dimensional plane. For any $\kappa$, we use $G^{(\kappa)}$ to denote the weighted complete graph over $P$ in which the weight of an edge $e$ is equal to $\|\mathbf{e}\|^{\kappa}$.

The minimum-energy unicast routing is essentially a shortest-path problem in $G^{(\kappa)}$. Consider any for a unicast from a node $\mathbf{p} \in \mathbf{P}$ to another node $\mathbf{q} \in \mathbf{P}$ can be easily obtained by applying a shortest-path algorithm to $G^{(\kappa)}$. Consider any unicast path from a node $\mathbf{p} \in \mathbf{P}$ to another node $\mathbf{q} \in \mathbf{P}$ :

$$
\mathbf{p}=\mathbf{p}_{\mathbf{0}} \mathbf{p}_{\mathbf{1}} \cdots \mathbf{p}_{\mathbf{m}-\mathbf{1}} \mathbf{p}_{\mathbf{m}}=\mathbf{q}
$$

In this path, the transmission power of node $\mathbf{p}_{\mathbf{i}}$ for $0 \leq$ $i \leq m-1$ is $\left\|\mathbf{p}_{\mathbf{i}} \mathbf{p}_{\mathbf{i}+\mathbf{1}}\right\|^{\kappa}$ and the transmission power of $\mathbf{p}_{\mathbf{m}}$ is zero. Thus the total transmission energy required by this path is

$$
\sum_{i=1}^{m-1}\left\|\mathbf{p}_{\mathbf{i}} \mathbf{p}_{\mathbf{i}+\mathbf{1}}\right\|^{\kappa},
$$

which is the total weight of this path. So by applying any shortest-path algorithm such as Dijkstra's algorithm [2], one can get a minimum-energy unicast routing.

However, for broadcast applications, and in general multicast applications, the minimum-energy routing is far more challenging. Any broadcast routing is an arborescence (a directed tree) $T$ rooted at the source node of the broadcasting that spans all nodes. We use $f_{T}(\mathbf{p})$ to denote the transmission power of the node $\mathbf{p}$ required by $T$. Then for any leaf node $\mathbf{p}$ of $T, f_{T}(\mathbf{p})=0$; and for any internal node $\mathbf{p}$ of $T$,

$$
f_{T}(\mathbf{p})=\max _{\mathbf{p q} \in \mathbf{T}}\|\mathbf{p q}\|^{\kappa},
$$

in other words, the $\kappa$-th power of the longest distance between $\mathbf{p}$ and its children in $T$. The total energy required by $T$ is then given by $\sum_{\mathbf{p} \in \mathbf{P}} f_{T}(\mathbf{p})$. Thus the minimum-energy broadcast routing is different from the conventional link-based minimum spanning tree (MST) problem. Indeed, while the MST can be solved in polynomial time by algorithms such as Prim's algorithm [2] and Kruskal's algorithm, it is still unknown whether minimum-energy broadcast routing can be solved in polynomial time. In its general graph version, the minimumenergy broadcast routing can be shown to be NP-hard [3], and even worse, inapproxiable within a factor of $(1-\epsilon) \log \Delta$, where $\Delta$ is the maximal degree and $\epsilon$ is any arbitrary small positive constant unless $N P \subseteq$ DTIME $\left[n^{O(\log \log n)}\right]$ by an approximation-preserving reduction from the Connected Dominating Set problem [4]. However, this intractness of its general graph version does not necessarily imply the same hardness of its geometric version. In fact, as will be shown later in the paper, its geometric version can be approximated within a constant factor. Nevertheless, this suggests that minimum-energy broadcast routing is considerably harder than the MST problem.

Three greedy heuristics have been proposed for the minimum-energy broadcast routing by [8]. The MST heuristic first applies the Prim's algorithm to obtain a MST, and then orient it as an arborescence rooted at the source node. The SPT heuristic first applies the Dijkstra's algorithm [2] to obtain a SPT, and then orient it 
as an arborescence rooted at the source node. The BIP heuristic is the node version of Dijkstra's algorithm for SPT. It maintains throughout its execution a single arborescence rooted at the source node. The arborescence starts from the source node, and new nodes are added to the arborescence one at a time on a minimum incremental cost basis until all nodes are included in the arborescence. The incremental cost of adding a new node is the minimum additional power increased by some node in the current arborescence so as to reach this new node. The implementation of BIP is based on the standard Dijkstra's algorithm, with one fundamental difference on the operation whenever a new node is added. Whereas Dijkstra's algorithm updates the node weights (representing the distances), BIP updates the link costs (representing the incremental power to reach the head node of the link). This update is performed by subtracting the cost of the added link from the cost of every link from the source node of the added link to a node not in new arborescence.

The performance of these three greedy heuristics have been evaluated in [8] by simulations studies. However, their analytic performances in terms of the approximation ratios remain open. The subsequent sections of this paper will derive the bounds on their approximation ratios.

\section{Greedy Is Not Always Good}

Greedy approaches are the most natural and widely used techniques in design practical heuristics for optimization problems. For minimum-energy broadcast routing, one may think of many greedy heuristics, in addition to the three greedy heuristics proposed in [8]. The real challenge, however, is how to come up with a provably good one. Two greedy criteria may only have slight difference, but these small variation can have a great impact on their performance. In addition, some criteria may perform quite well or even optimally in some situations, but may perform very poorly in some other situations. In this section, we describe two examples, one is SPT, another is a new one. The "bad" instance constructed in this section can not only lead to lower bounds on their approximation ration, but also help to design an overall good greedy criteria. For the simplicity, we only consider $\kappa=2$ in this section.

We begin with the SPT algorithm. Let $\epsilon$ be a sufficiently small positive number. Consider $m$ nodes $\mathbf{p}_{\mathbf{1}}, \mathbf{p}_{\mathbf{2}}, \cdots, \mathbf{p}_{\mathbf{m}}$ evenly distributed on a cycle of radius 1 centered at node o (see Figure 2). For $1 \leq i \leq m$, let $\mathbf{q}_{\mathbf{i}}$ be the point in the line segment $\mathbf{o p}_{\mathbf{i}}$ with $\left\|\mathbf{o q}_{\mathbf{i}}\right\|=\epsilon$. We consider a broadcasting from node $\mathbf{o}$ to these $n=2 m$ nodes

$$
\mathbf{p}_{1}, \mathbf{p}_{2}, \cdots, \mathbf{p}_{\mathbf{m}}, \mathbf{q}_{1}, \mathbf{q}_{2}, \cdots, \mathbf{q}_{\mathbf{m}} .
$$

The SPT is the superposition of the paths $\mathbf{o} \mathbf{q}_{\mathbf{i}} \mathbf{p}_{\mathbf{i}}$. Its total energy is

$$
\epsilon^{2}+m(1-\epsilon)^{2}
$$

On the other hand, if the transmission power of node $\mathbf{o}$ is 1 , then the signal can reach all other points. Thus the minimum energy is at most 1 . So the approximation ratio of SPT is $\epsilon^{2}+m(1-\epsilon)^{2}$. As $\epsilon \longrightarrow 0$, this ratio converges to $\frac{n}{2}=m$.

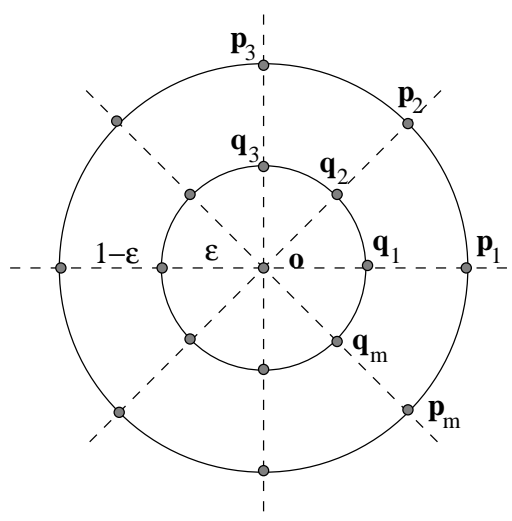

Fig. 2. A bad instance for SPT.

The second greedy heuristic is very similar to Chvatal's algorithm [1] for Set Cover Problem and is a variation of BIP. Like BIP, an arborescence, which starts with the source node, is maintained throughout the execution of algorithm. However, unlike BIP, many new nodes can be added one time. Similar to Chvatal's algorithm [1], the new nodes added are chosen to have the minimal average incremental cost, which is defined as the ratio of the minimum addition power increased by some node in the current arborescence so as to reach these new nodes to the number of these new nodes. We refer to this heuristic as the Broadcast Average Incremental Power, abbreviated by BAIP. In contrast to the $1+\log m$ approximation ratio of the Chvatal's algorithm [1] where $m$ is the largest set size, we show that the approximation ratio of BAIP is at least $\frac{4 n}{\ln n}-o(1)$ where $n$ is the number of receiving nodes.

Consider the following instance of minimum-energy broadcasting. All nodes are colinear with the source being the origin, the $i$-th receiving node at the position $\sqrt{i}$ for $1 \leq i \leq n-1$, and the $n$-th receiving node at the position $\sqrt{n-\epsilon}$ for some sufficiently small number $\epsilon>0$. For any $1 \leq k \leq n-1$, the minimal transmission power of the source to reach $k$ receiving nodes is $(\sqrt{k})^{2}=k$, and thus the average power efficiency is $\frac{k}{k}=1$. On the other hand, the minimal transmission power of the source to reach all $n$ receiving nodes is $(\sqrt{n-\epsilon})^{2}=n-\epsilon$, and the thus the average power efficiency is $\frac{n-\epsilon}{n}=1-\frac{\epsilon}{n}$. So BAIP will let the source to transmit at power $n-\epsilon$ to reach all nodes in a single step. However, the optimal routing is a directed path consisting of all nodes from left 
to right. So the minimum power is

$$
\begin{aligned}
& \sum_{i=1}^{n-1}(\sqrt{i}-\sqrt{i-1})^{2}+(\sqrt{n-\epsilon}-\sqrt{n-1})^{2} . \\
& <\sum_{i=1}^{n}(\sqrt{i}-\sqrt{i-1})^{2}=1+\sum_{i=1}^{n-1} \frac{1}{(\sqrt{i+1}+\sqrt{i})^{2}} \\
& \leq 1+\sum_{i=1}^{n-1} \frac{1}{4 i} \leq 1+\frac{\ln (n-1)+1}{4} \\
& =\frac{\ln (n-1)+5}{4} .
\end{aligned}
$$

Thus the approximation ratio is at least

$$
\frac{n-\epsilon}{\sum_{i=1}^{n-1}(\sqrt{i}-\sqrt{i-1})^{2}+(\sqrt{n-\epsilon}-\sqrt{n-1})^{2}} .
$$

As $\epsilon \longrightarrow 0$, this ratio converges to

$$
\begin{aligned}
& \frac{n}{\sum_{i=1}^{n}(\sqrt{i}-\sqrt{i-1})^{2}}=\frac{n}{1+\sum_{i=1}^{n-1} \frac{1}{(\sqrt{i+1}+\sqrt{i})^{2}}} \\
& \geq \frac{n}{1+\sum_{i=1}^{n-1} \frac{1}{4 i}} \geq \frac{n}{1+\frac{\ln (n-1)+1}{4}} \\
& =\frac{4 n}{\ln (n-1)+5}=\frac{4 n}{\ln n}-o(1)
\end{aligned}
$$

Interestingly, SPT generates the optimal solution in the second bad instance, while BAIP can provide nearoptimal or optimal solution for the first bad instance. On the other hand, MST and BIP have a lot similarities to SPT and BAIP, but have constant approximation ratios as will be proved later. Thus one should carefully design and select greedy heuristics.

\section{Lower Bounds on Approximation Ratios}

In this section, we will derive lower bounds on approximation ratios of MST and BIP. We begin with MST.

Theorem 1: The approximation ratio of MST is at least 6 for any $\kappa \geq 2$.

Proof: Let $\epsilon$ be a sufficiently small positive number. Consider seven nodes $\mathbf{o}, \mathbf{p}_{\mathbf{1}}, \cdots, \mathbf{p}_{\mathbf{6}}$ (see Figure 3 ), which satisfies that

$$
\begin{aligned}
\left\|\mathbf{o} \mathbf{p}_{\mathbf{1}}\right\| & =1 \\
\left\|\mathbf{o p}_{\mathbf{i}}\right\| & =1+\epsilon, 2 \leq i \leq 6 ; \\
\left\|\mathbf{p}_{\mathbf{i}} \mathbf{p}_{\mathbf{i}+\mathbf{1}}\right\| & =1,1 \leq i \leq 5 .
\end{aligned}
$$

Then for any $1 \leq i \leq 5$,

$$
\angle \mathbf{p}_{\mathbf{i}} \mathbf{p p}_{\mathbf{i}+\mathbf{1}}<\frac{\pi}{3}
$$

and

$$
\angle \mathbf{p}_{6} \mathbf{o p}_{1}>\frac{\pi}{3}
$$

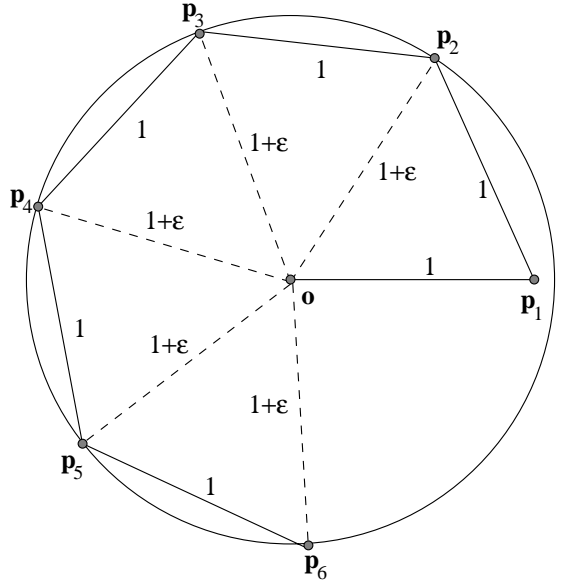

Fig. 3. A bad instance for MST.

Consider the two triangles $\mathbf{o p}_{\mathbf{1}} \mathbf{p}_{\mathbf{2}}$ and $\mathbf{o p}_{\mathbf{1}} \mathbf{p}_{\mathbf{6}}$. Since

$$
\left\|\mathrm{op}_{2}\right\|=\left\|\mathrm{op}_{\mathbf{6}}\right\|
$$

and

$$
\angle \mathbf{p}_{6} \mathbf{o p}_{1}>\angle \mathbf{p}_{1} \mathbf{o p}_{2},
$$

by Law of Cosine, we have

$$
\left\|\mathbf{p}_{\mathbf{1}} \mathbf{p}_{\mathbf{6}}\right\|>\left\|\mathbf{p}_{\mathbf{1}} \mathbf{p}_{\mathbf{2}}\right\|=1 \text {. }
$$

We consider the broadcasting from the node $\mathbf{o}$ to nodes $\mathbf{p}_{1}, \cdots, \mathbf{p}_{\mathbf{6}}$. Then the path $\mathbf{o p}_{\mathbf{1}} \cdots \mathbf{p}_{\mathbf{5}} \mathbf{p}_{\mathbf{6}}$ is the unique MST. It's total energy is 6 . On the other hand, the optimal routing is the star centered at node o, whose total energy is $(1+\epsilon)^{\kappa}$. Thus the approximation ratio is at least $\frac{6}{(1+\epsilon)^{\kappa}}$, which converges to 6 as $\epsilon \longrightarrow 0$.

Now we develop the lower bound on the approximation ratio of BIP.

Theorem 2: The approximation ratio of BIP is at least $\frac{13}{3}$ for any $\kappa=2$.

Proof: Let $\theta$ be a sufficiently small positive number. Consider six points $\mathbf{p}_{\mathbf{1}}, \cdots, \mathbf{p}_{\mathbf{6}}$ on a cycle of radius 1 centered at node $\mathbf{o}$ (see Figure 4 ), with

$$
\begin{aligned}
& \angle \mathbf{p}_{1} \mathbf{o p}_{\mathbf{2}}=\angle \mathbf{p}_{\mathbf{5}} \mathbf{o} \mathbf{p}_{\mathbf{6}}=\frac{\pi}{\mathbf{3}}-\mathbf{3} \theta \\
& \angle \mathbf{p}_{\mathbf{2}} \mathbf{o p}_{\mathbf{3}}=\angle \mathbf{p}_{\mathbf{4}} \mathbf{o} \mathbf{p}_{\mathbf{5}}=\frac{\pi}{\mathbf{3}}-\mathbf{2} \theta \\
& \angle \mathbf{p}_{\mathbf{3}} \mathbf{o} \mathbf{p}_{\mathbf{4}}=\frac{\pi}{3}-\theta \\
& \angle \mathbf{p}_{\mathbf{6}} \mathbf{o p}_{\mathbf{1}}=\frac{\pi}{3}+11 \theta
\end{aligned}
$$

Then

$$
\begin{aligned}
&\left\|\mathbf{p}_{\mathbf{1}} \mathbf{p}_{\mathbf{2}}\right\|=\left\|\mathbf{p}_{\mathbf{5}} \mathbf{p}_{\mathbf{6}}\right\|< \\
&\left\|\mathbf{p}_{\mathbf{2}} \mathbf{p}_{\mathbf{3}}\right\|=\left\|\mathbf{p}_{\mathbf{4}} \mathbf{p}_{\mathbf{5}}\right\|< \\
&\left\|\mathbf{p}_{\mathbf{3}} \mathbf{p}_{\mathbf{4}}\right\|<1<\left\|\mathbf{p}_{\mathbf{6}} \mathbf{p}_{\mathbf{1}}\right\| .
\end{aligned}
$$




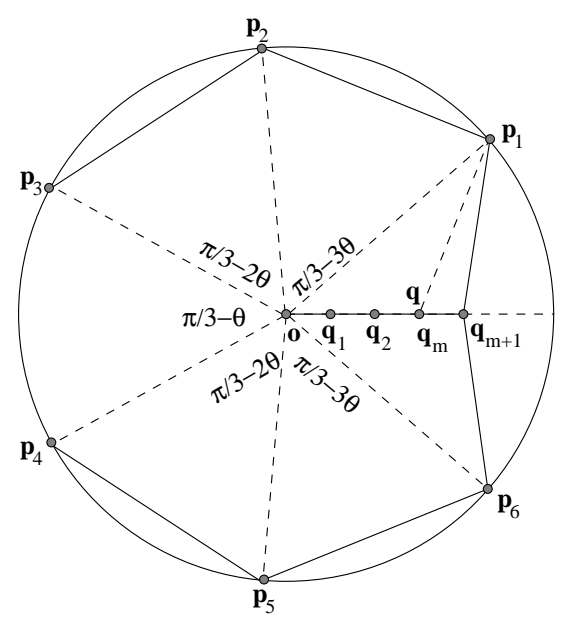

Fig. 4. A bad instance for BIP.

Let $\mathbf{q}$ be the point in the perpendicular bisector of $\mathbf{p}_{\mathbf{1}} \mathbf{p}_{\mathbf{6}}$ such that $\mathbf{p}_{\mathbf{1}} \mathbf{q}$ is perpendicular to $\mathbf{p}_{\mathbf{1}} \mathbf{p}_{\mathbf{2}}$. Choose a sufficiently large integer $m$ such that

$$
1-\left(\frac{\|\mathbf{o q}\|}{m}\right)^{2}>\left\|\mathbf{p}_{3} \mathbf{p}_{4}\right\|^{2} .
$$

Let $\mathbf{q}_{\mathbf{1}}, \cdots, \mathbf{q}_{\mathbf{m}+\mathbf{1}}$ be the $m+1$ points on the ray oq with

$$
\left\|\mathbf{o q}_{\mathbf{i}}\right\|=\frac{i}{m}\|\mathbf{o q}\|
$$

for $1 \leq i \leq m+1$. Then $\mathbf{q}_{\mathbf{m}}=\mathbf{q}$.

We consider a broadcasting from point $\mathbf{o}$ to points $\mathbf{q}_{\mathbf{1}}, \cdots, \mathbf{q}_{\mathbf{m}+\mathbf{1}}, \mathbf{p}_{\mathbf{1}}, \cdots, \mathbf{p}_{\mathbf{6}}$. The optimal solution is that the node o transmits at power 1 to reach all nodes. Now let's examine the output of the BIP algorithm. As $m$ is sufficiently large, in the first $m+1$ steps, the points $\mathbf{q}_{\mathbf{1}}, \cdots, \mathbf{q}_{\mathbf{m}+\mathbf{1}}$ are sequentially added, and the transmission power of the nodes $\mathbf{o}, \mathbf{q}_{\mathbf{1}}, \cdots, \mathbf{q}_{\mathbf{m}}$ all has the transmission power $\left(\frac{\|\mathbf{o q}\|}{m}\right)^{2}$. Since the angles

$$
\angle \mathbf{p}_{\mathbf{1}} \mathbf{q}_{\mathbf{m}+\mathbf{1}} \mathbf{q}_{\mathbf{m}}=\angle \mathbf{p}_{\mathbf{6}} \mathbf{q}_{\mathbf{m}+\mathbf{1}} \mathbf{q}_{\mathbf{m}}>\frac{\pi}{\mathbf{2}},
$$

in the next two steps, the points $\mathbf{p}_{\mathbf{1}}$ and $\mathbf{p}_{\mathbf{6}}$ are added, and the transmission power of point $\mathbf{q}_{\mathbf{m}+\mathbf{1}}$ is $\left\|\mathbf{p}_{\mathbf{1}} \mathbf{q}_{\mathbf{m}+\mathbf{1}}\right\|^{2}$. At this moment, the incremental power of all points $\mathbf{o}, \mathbf{q}_{\mathbf{1}}, \cdots, \mathbf{q}_{\mathbf{m}}$ to reach any node $\mathbf{p}_{\mathbf{i}}$ for $2 \leq i \leq 5$ is at least

$$
1-\left(\frac{\|\mathbf{o q}\|}{m}\right)^{2}>\left\|\mathbf{p}_{\mathbf{3}} \mathbf{p}_{\mathbf{4}}\right\|^{2}>\left\|\mathbf{p}_{\mathbf{1}} \mathbf{p}_{\mathbf{2}}\right\|^{2}=\left\|\mathbf{p}_{\mathbf{5}} \mathbf{p}_{\mathbf{6}}\right\|^{2},
$$

and the incremental power of point $\mathbf{q}_{\mathbf{m}+\mathbf{1}}$ to reach any node $\mathbf{p}_{\mathbf{i}}$ for $2 \leq i \leq 5$ is also greater than $\left\|\mathbf{p}_{\mathbf{1}} \mathbf{p}_{\mathbf{2}}\right\|^{2}=$ $\left\|\mathbf{p}_{5} \mathbf{p}_{\mathbf{6}}\right\|^{2}$ as

$$
\angle \mathbf{p}_{2} \mathbf{p}_{1} \mathbf{q}_{\mathbf{m}+1}=\angle \mathbf{p}_{5} \mathbf{p}_{6} \mathbf{q}_{\mathrm{m}+1}>\angle \mathbf{p}_{2} \mathbf{p}_{1} \mathbf{q}_{\mathrm{m}}=\frac{\pi}{2} .
$$

Thus in the subsequent two steps, the points $\mathbf{p}_{\mathbf{2}}$ and $\mathbf{p}_{\mathbf{5}}$ are added, and the transmission power of points $\mathbf{p}_{\mathbf{1}}$ and $\mathbf{p}_{\mathbf{6}}$ is $\left\|\mathbf{p}_{\mathbf{1}} \mathbf{p}_{\mathbf{2}}\right\|^{2}=\left\|\mathbf{p}_{\mathbf{5}} \mathbf{p}_{\mathbf{6}}\right\|^{2}$. Similarly, in the last two steps, the points $\mathbf{p}_{\mathbf{3}}$ and $\mathbf{p}_{\mathbf{4}}$ are added, and the transmission power of points $\mathbf{p}_{\mathbf{2}}$ and $\mathbf{p}_{5}$ is $\left\|\mathbf{p}_{\mathbf{2}} \mathbf{p}_{\mathbf{3}}\right\|^{2}=\left\|\mathbf{p}_{4} \mathbf{p}_{5}\right\|^{2}$. The total power is

$$
\begin{aligned}
& (m+1)\left(\frac{\|\mathbf{o q}\|}{m}\right)^{2}+\left\|\mathbf{p}_{\mathbf{1}} \mathbf{q}_{\mathbf{m}+\mathbf{1}}\right\|^{2}+2\left\|\mathbf{p}_{\mathbf{1}} \mathbf{p}_{\mathbf{2}}\right\|^{2}+2\left\|\mathbf{p}_{\mathbf{2}} \mathbf{p}_{\mathbf{3}}\right\|^{2} \\
& =\frac{m+1}{m^{2}}\|\mathbf{o q}\|^{2}+\left\|\mathbf{p}_{\mathbf{1}} \mathbf{q}_{\mathbf{m}+\mathbf{1}}\right\|^{2}+2\left\|\mathbf{p}_{\mathbf{1}} \mathbf{p}_{\mathbf{2}}\right\|^{2}+2\left\|\mathbf{p}_{\mathbf{2}} \mathbf{p}_{\mathbf{3}}\right\|^{2} .
\end{aligned}
$$

As $\theta \longrightarrow 0$ and $m \longrightarrow \infty$, the polygon $\mathbf{p}_{\mathbf{1}} \mathbf{p}_{\mathbf{2}} \mathbf{p}_{\mathbf{3}} \mathbf{p}_{\mathbf{4}} \mathbf{p}_{\mathbf{5}} \mathbf{p}_{\mathbf{6}}$ converges to a regular hexagon, and the nodes $\mathbf{q}$ and $\mathbf{q}_{\mathbf{m}+\mathbf{1}}$ converges to the center of the triangle $\mathbf{o p}_{\mathbf{1}} \mathbf{p}_{\mathbf{6}}$. Thus the total power converges to $\frac{1}{3}+4=\frac{13}{3}$. Thus the approximation ratio of BIP is at least $\frac{13}{3} \approx 4.33$.

\section{Upper Bounds on Approximation Ratios}

Our deriving of the upper bounds relies on extensively on the geometric structures of Euclidean MSTs. We first observe that as long as the cost of a link is an increasing function of the Euclidean length of the link, the set of MSTs of any point set coincides with the set of Euclidean MSTs of the same point set. In fact, this can be followed from Prim's algorithm. In particular, for any spanning tree $T$ of a (finite) point set $P$, the parameter $\sum_{e \in T}\|e\|^{2}$ achieves its minimum if and only if $T$ is an Euclidean MST of $P$. For any (finite) point set $P$, we use $m s t(P)$ to denote an arbitrary Euclidean MST of $P$. The radius of a point set $P$ is defined as

$$
\inf _{\mathbf{p} \in \mathbf{P}} \sup _{\mathbf{q} \in \mathbf{P}}\|\mathbf{p q}\| \text {. }
$$

Thus a point set of radius one can be covered by a disk of the same radius. A key result in this section is an upper bound on the parameter $\sum_{e \in m s t(P)}\|e\|^{2}$ for any finite point set $P$ of radius one. Note that the supreme of the length of $m s t(P), \sum_{e \in m s t(P)}\|e\|$, over all point sets $P$ of radius one is infinity. Amazingly however, the parameter $\sum_{e \in m s t(P)}\|e\|^{2}$ is bounded by a constant for any point set $P$ of radius one, as shown later. We use $c$ to denote the supreme of the length of $\sum_{e \in m s t(P)}\|e\|^{2}$ over all point sets $P$ of radius one. The next key theorem states that $c$ is at most 12 .

Theorem 3: $6 \leq c \leq 12$.

The proof of this theorem involves complicated geometric arguments, and therefore we postpone it in Section VI. Note that for any point set $P$ of radius one, the length of each edge in $m s t(P)$ is at most one. Therefore, Theorem 3 implies that for any point set $P$ of radius one and any $\kappa \geq 2$,

$$
\sum_{e \in m s t(P)}\|e\|^{\kappa} \leq \sum_{e \in m s t(P)}\|e\|^{2} \leq c \leq 12 .
$$


In the next, we explore a relation between the minimum energy required by a broadcasting and the Euclidean MST of the corresponding point set.

Lemma 4: For any point set $P$ in the plane, the total anergy required by any broadcasting among $P$ is at least $\frac{1}{c} \sum_{e \in m s t(P)}\|e\|^{\kappa}$.

Proof: $\quad$ Let $T$ be an optimal arborescence for a broadcasting among $P$. For any none-leaf node $\mathbf{p}$ in $T$, let $T_{\mathbf{p}}$ be an Euclidean MST of the point set consisting $\mathbf{p}$ and all children of $\mathbf{p}$ in $T$. Suppose that the longest Euclidean distance between $\mathbf{p}$ and its children is $r$. Then the transmission power of node $\mathbf{p}$ is $r^{\kappa}$, and all children of $\mathbf{p}$ lie in the disk centered at $\mathbf{p}$ with radius $r$. From the definition of $c$, we have

$$
\sum_{e \in T_{\mathbf{p}}}\left(\frac{\|e\|}{r}\right)^{\kappa} \leq c
$$

which implies that

$$
r^{\kappa} \geq \frac{1}{c} \sum_{e \in T_{\mathbf{p}}}^{\kappa}\|e\|^{\kappa}
$$

Let $T^{*}$ denote the spanning tree obtained by superposing of all $T_{\mathbf{p}}$ 's for non-leaf nodes of $T$. Then the total energy required by $T$ is at least $\frac{1}{c} \sum_{e \in T^{*}}\|e\|^{\kappa}$, which is further no less than $\frac{1}{c} \sum_{e \in m s t(P)}\|e\|^{\kappa}$. This completes the proof.

Consider any point set $P$ in the plane. Let $T$ be an arborescence oriented from some $m s t(P)$. Then the total energy required by $T$ is at most $\sum_{e \in T_{\mathbf{p}}}^{\kappa}\|e\|^{\kappa}$. From Lemma 4, this total energy is at most $c$ times the optimum cost. Thus the approximation ratio of MST is at most $c$. Together with Theorem 3 , this observation leads to the following theorem.

Theorem 5: The approximation ratio of MST is at most $c$, and therefore is at most 12 .

Finally, we derive the upper bound on the approximation ratio of the BIP heuristic. Once again, the Euclidean MST will play an important role.

Lemma 6: For any broadcasting among a point set $P$ in the plane, the total energy required by the arborescence generated by BIP algorithm is at most $\sum_{e \in m s t(P)}\|e\|^{\kappa}$.

Proof: Let $G^{(\kappa)}$ be the complete graph over the point set $P$, in which the weight of an edge $e$ is $\|e\|^{\kappa}$. Let $T$ be the arborescence output by the algorithm BIP. We construct another weighted graph $H$ over the same point set $P$ according to the execution of BIP for generating $T$. Suppose that during the execution of BIP the nodes are added in the order $\mathbf{p}_{\mathbf{1}}, \mathbf{p}_{\mathbf{2}}, \cdots, \mathbf{p}_{\mathbf{n}}$ where $\mathbf{p}_{\mathbf{1}}$ is the source node. Let $T_{i}$ be the arborescence just after node $\mathbf{p}_{\mathbf{i}}$ is added. In $H$, the weight of the edge $\mathbf{p}_{\mathbf{i}} \mathbf{p}_{\mathbf{i}+\mathbf{1}}$ is equal to the incremental energy of the link from $T_{i}$ to $\mathbf{p}_{\mathbf{i}+\mathbf{1}}$ chosen during the execution of SPF; and the weight of any other edge is the same as that in $G^{(\kappa)}$. Note that for each edge $\mathbf{p}_{\mathbf{i}} \mathbf{p}_{\mathbf{i}+\mathbf{1}}$, its weight in $H$ is not more than its weight in
$G^{(\kappa)}$. Therefore, for any spanning tree, its weight in $H$ is no more than its weight in $G^{(\kappa)}$. On the other hand, the execution of Prim's algorithm on $H$ will emulate the algorithm BIP on $G^{(\kappa)}$ in the sense that it will add the required nodes in the same order, and will output the path $\mathbf{p}_{\mathbf{1}} \mathbf{p}_{\mathbf{2}} \cdots \mathbf{p}_{\mathbf{n}}$. The weight of this path in $H$ is exactly the total energy required by $T$, but is at most the weight of any MST in $G^{(\kappa)}$. This implies that the total energy required by $T$ is at most $\sum_{e \in m s t(P)}\|e\|^{\kappa}$. This completes the proof.

From the above lemma and Lemma 4, we can get the result for the BIP algorithm similar to Theorem 5 .

Theorem 7: The approximation ratio of the BIP heuristic is at most $c$, and therefore is at most 12 .

\section{Proof of Theorem 3}

This section is devoted to the proof of Theorem 3. The lower bound is very trivial as it can follow from the instance consisting of seven points: the center of a regular hexagon and its six vertices. However, the deriving of the upper bound is very challenging. We first introduce some geometric structures and notations to be used in this section. All angles are measured in radians and take values in the range $[0, \pi]$. For any three points $\mathbf{p}_{\mathbf{1}}, \mathbf{p}_{\mathbf{2}}$ and $\mathbf{p}_{\mathbf{3}}$, the angle between the two rays $\mathbf{p}_{\mathbf{1}} \mathbf{p}_{\mathbf{2}}$ and $\mathbf{p}_{\mathbf{1}} \mathbf{p}_{\mathbf{3}}$ is denoted by $\angle \mathbf{p}_{\mathbf{2}} \mathbf{p}_{\mathbf{1}} \mathbf{p}_{\mathbf{3}}$ or $\angle \mathbf{p}_{\mathbf{3}} \mathbf{p}_{\mathbf{1}} \mathbf{p}_{\mathbf{2}}$. The closed infinite area inside the angle $\angle \mathbf{p}_{\mathbf{2}} \mathbf{p}_{\mathbf{1}} \mathbf{p}_{\mathbf{3}}$, also referred to as a sector, is denoted by $\measuredangle \mathbf{p}_{\mathbf{2}} \mathbf{p}_{\mathbf{1}} \mathbf{p}_{\mathbf{3}}$. The triangle determined $\mathbf{p}_{\mathbf{1}}, \mathbf{p}_{\mathbf{2}}$ and $\mathbf{p}_{\mathbf{3}}$ is denoted by $\triangle \mathbf{p}_{\mathbf{2}} \mathbf{p}_{\mathbf{1}} \mathbf{p}_{\mathbf{3}}$. The open disk centered at $\mathbf{p}$ with radius $r$, denoted by $\mathbf{B}(\mathbf{p}, \mathbf{r})$, is the set of points whose distance from $\mathbf{p}$ is less than $r$. The lune through points $\mathbf{p}_{\mathbf{1}}$ and $\mathbf{p}_{\mathbf{2}}$, denoted by $L\left(\mathbf{p}_{\mathbf{1}} \mathbf{p}_{\mathbf{2}}\right)$, is the intersection of the two open disks of radius $\left\|\mathbf{p}_{\mathbf{1}} \mathbf{p}_{\mathbf{2}}\right\|$ centered at $\mathbf{p}_{1}$ and $\mathbf{p}_{2}$ respectively (see Figure 5(a)). Thus it consists of points whose distances from $\mathbf{p}_{\mathbf{1}}$ and $\mathbf{p}_{\mathbf{2}}$ are both less than $\left\|\mathbf{p}_{\mathbf{1}} \mathbf{p}_{\mathbf{2}}\right\|$. The open diamond substended by a line segment $\mathbf{p}_{\mathbf{1}} \mathbf{p}_{\mathbf{2}}$, denoted by $\mathbf{D}\left(\mathbf{p}_{\mathbf{1}} \mathbf{p}_{\mathbf{2}}\right)$, is the rhombus with sides of length $\frac{\sqrt{3}}{3}\left\|\mathbf{p}_{\mathbf{1}} \mathbf{p}_{\mathbf{2}}\right\|$ (see Figure $5(\mathrm{~b})$ ). Note that the interior angles at $\mathbf{p}_{\mathbf{1}}$ and $\mathbf{p}_{\mathbf{2}}$ within $\mathbf{D}\left(\mathbf{p}_{\mathbf{1}} \mathbf{p}_{\mathbf{2}}\right)$ are equal to $\frac{\pi}{3}$.

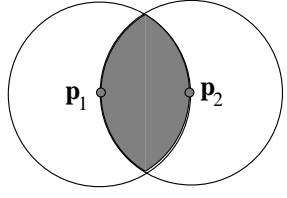

(a)

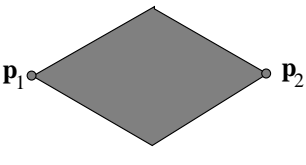

(b)
Fig. 5. Illustration of (a) lune and (b) diamond.

The Euclidean MSTs have many nice structure properties [5]. Some basic properties are listed blow.

- Any pair of edges do not cross each other.

- The angles between any two edges incident to a common vertex is at least $\frac{\pi}{3}$. 
- The length of each edge is at most the radius of the vertex set.

- The luna determined by each edge does not contain any other vertices.

- Let $\mathbf{p}_{\mathbf{1}} \mathbf{p}_{\mathbf{2}}$ be any edge. Then the two endpoints of any other edge are either both outside $\mathbf{B}\left(\mathbf{p}_{\mathbf{1}},\left\|\mathbf{p}_{\mathbf{1}} \mathbf{p}_{\mathbf{2}}\right\|\right)$, or both outside $\mathbf{B}\left(\mathbf{p}_{\mathbf{2}},\left\|\mathbf{p}_{\mathbf{1}} \mathbf{p}_{\mathbf{2}}\right\|\right)$.

In this section, we will first prove yet another structure property of the Euclidean MSTs, which is very essential to bound the constant $c$ : The diamonds of any two edges are disjoint. The proof of this property will make use of the following lemma.

Lemma 8: Let $\mathbf{p}_{\mathbf{1}}, \mathbf{p}_{\mathbf{2}}$ and $\mathbf{p}_{\mathbf{3}}$ be any three points in the plane with $\angle \mathbf{p}_{\mathbf{1}} \mathbf{p}_{\mathbf{3}} \mathbf{p}_{\mathbf{2}}=\frac{\mathbf{2} \pi}{\mathbf{3}}$ and $\left\|\mathbf{p}_{\mathbf{1}} \mathbf{p}_{\mathbf{3}}\right\|=\left\|\mathbf{p}_{\mathbf{2}} \mathbf{p}_{\mathbf{3}}\right\|$ (see Figure 6). Let $\mathbf{p}_{\mathbf{4}}$ be any point in $\measuredangle \mathbf{p}_{\mathbf{1}} \mathbf{p}_{\mathbf{3}} \mathbf{p}_{\mathbf{2}}$ but outside $\triangle \mathbf{p}_{\mathbf{1}} \mathbf{p}_{\mathbf{2}} \mathbf{p}_{\mathbf{3}}$ with $\angle \mathbf{p}_{\mathbf{2}} \mathbf{p}_{\mathbf{1}} \mathbf{p}_{\mathbf{4}}=\alpha$. Then $\mathbf{D}\left(\mathbf{p}_{\mathbf{1}} \mathbf{p}_{\mathbf{4}}\right) \subseteq$ $\measuredangle \mathbf{p}_{\mathbf{1}} \mathbf{p}_{\mathbf{3}} \mathbf{p}_{\mathbf{2}}$ if and only if either $\alpha \in\left[0, \frac{\pi}{3}\right)$ and $\left\|\mathbf{p}_{\mathbf{1}} \mathbf{p}_{\mathbf{4}}\right\| \leq$ $\frac{\sin \frac{\pi}{3}}{\sin \left(\frac{\pi}{3}-\alpha\right)}\left\|\mathbf{p}_{\mathbf{1}} \mathbf{p}_{\mathbf{2}}\right\|$ or $\alpha \in\left[\frac{\pi}{3}, \frac{5 \pi}{6}\right]$.

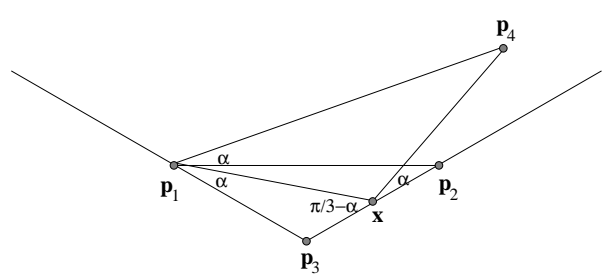

(a)

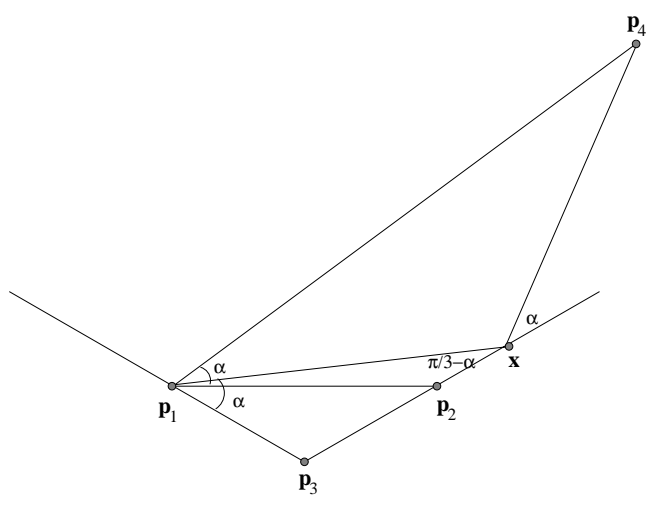

(b)

Fig. 6. Two extreme cases for $\mathbf{D}\left(\mathbf{p}_{\mathbf{1}} \mathbf{p}_{\mathbf{4}}\right) \subseteq \measuredangle \mathbf{p}_{\mathbf{1}} \mathbf{p}_{\mathbf{3}} \mathbf{p}_{\mathbf{2}}$.

Proof: $\quad$ Note that $\mathbf{D}\left(\mathbf{p}_{\mathbf{1}} \mathbf{p}_{\mathbf{4}}\right) \varsubsetneqq \measuredangle \mathbf{p}_{\mathbf{1}} \mathbf{p}_{\mathbf{3}} \mathbf{p}_{\mathbf{2}}$ if $\alpha>\frac{5 \pi}{6}$; and $\mathbf{D}\left(\mathbf{p}_{\mathbf{1}} \mathbf{p}_{\mathbf{4}}\right) \subseteq \measuredangle \mathbf{p}_{\mathbf{1}} \mathbf{p}_{\mathbf{3}} \mathbf{p}_{\mathbf{2}}$ if $\alpha \in\left[\frac{\pi}{3}, \frac{5 \pi}{6}\right]$. So we now assume $\alpha \in\left[0, \frac{\pi}{3}\right)$. We fix $\alpha$ and calculate the maximum length of $\mathbf{p}_{\mathbf{1}} \mathbf{p}_{\mathbf{4}}$ such that $\mathbf{D}\left(\mathbf{p}_{\mathbf{1}} \mathbf{p}_{\mathbf{4}}\right) \subseteq \measuredangle \mathbf{p}_{\mathbf{1}} \mathbf{p}_{\mathbf{3}} \mathbf{p}_{\mathbf{2}}$. This happens when $\mathbf{D}\left(\mathbf{p}_{\mathbf{1}} \mathbf{p}_{\mathbf{4}}\right)$ touches the ray $\mathbf{p}_{\mathbf{3}} \mathbf{p}_{\mathbf{2}}$, say at $\mathbf{x}$. We consider this extreme scenario. In this case,

$$
\angle \mathbf{p}_{\mathbf{3}} \mathbf{p}_{\mathbf{1}} \mathbf{x}=\alpha, \angle \mathbf{p}_{\mathbf{1}} \mathbf{x p}_{\mathbf{3}}=\frac{\pi}{\mathbf{3}}-\alpha .
$$

Applying the Laws of Sine in $\triangle \mathbf{p}_{\mathbf{1}} \mathbf{p}_{\mathbf{3}} \mathbf{x}$, we have

$$
\frac{\left\|\mathbf{p}_{\mathbf{1}} \mathbf{x}\right\|}{\left\|\mathbf{p}_{\mathbf{1}} \mathbf{p}_{\mathbf{3}}\right\|}=\frac{\sin \frac{\pi}{3}}{\sin \left(\frac{\pi}{3}-\alpha\right)} \text {. }
$$

On the other hand, as $\triangle \mathbf{p}_{\mathbf{1}} \mathbf{p}_{\mathbf{2}} \mathbf{p}_{\mathbf{3}}$ and $\triangle \mathbf{p}_{\mathbf{1}} \mathbf{p}_{\mathbf{4}} \mathbf{x}$ are similar,

$$
\frac{\left\|\mathbf{p}_{\mathbf{1}} \mathbf{p}_{\mathbf{4}}\right\|}{\left\|\mathbf{p}_{\mathbf{1}} \mathbf{p}_{\mathbf{2}}\right\|}=\frac{\left\|\mathbf{p}_{\mathbf{1}} \mathbf{x}\right\|}{\left\|\mathbf{p}_{\mathbf{1}} \mathbf{p}_{\mathbf{3}}\right\|}=\frac{\sin \frac{\pi}{3}}{\sin \left(\frac{\pi}{3}-\alpha\right)}
$$

Therefore, $\mathbf{D}\left(\mathbf{p}_{\mathbf{1}} \mathbf{p}_{\mathbf{4}}\right) \subseteq \measuredangle \mathbf{p}_{\mathbf{1}} \mathbf{p}_{\mathbf{3}} \mathbf{p}_{\mathbf{2}}$ as long as $\left\|\mathbf{p}_{\mathbf{1}} \mathbf{p}_{\mathbf{4}}\right\| \leq$ $\frac{\sin \frac{\pi}{3}}{\sin \left(\frac{\pi}{3}-\alpha\right)}\left\|\mathbf{p}_{\mathbf{1}} \mathbf{p}_{\mathbf{2}}\right\|$.

In the next we apply the above lemma to show that the diamond determined by any edge in an Euclidean MST is contained in some sector defined in the next lemma.

Lemma 9: Let $\mathbf{p}_{\mathbf{1}}, \mathbf{p}_{\mathbf{2}}$ and $\mathbf{p}_{\mathbf{3}}$ be any three points in the plane with $\mathbf{p}_{\mathbf{3}}$ being outside $L\left(\mathbf{p}_{\mathbf{1}} \mathbf{p}_{\mathbf{2}}\right)$. Let $\mathbf{p}_{\mathbf{1}}^{\prime}\left(\mathbf{p}_{\mathbf{2}}^{\prime}\right.$ respectively) be the vertex of $\mathbf{D}\left(\mathbf{p}_{\mathbf{1}} \mathbf{p}_{\mathbf{3}}\right)\left(\mathbf{D}\left(\mathbf{p}_{\mathbf{2}} \mathbf{p}_{\mathbf{3}}\right)\right.$ respectively) which is lies on the opposite side of the line $\mathbf{p}_{\mathbf{1}} \mathbf{p}_{\mathbf{3}}\left(\mathbf{p}_{\mathbf{2}} \mathbf{p}_{\mathbf{3}}\right.$ respectively) from $\mathbf{p}_{\mathbf{2}}\left(\mathbf{p}_{\mathbf{1}}\right.$ respectively) (see Figure 7 ). Then $\mathbf{D}\left(\mathbf{p}_{\mathbf{1}} \mathbf{p}_{\mathbf{2}}\right) \subseteq \measuredangle \mathbf{p}_{\mathbf{1}}^{\prime} \mathbf{p}_{\mathbf{3}} \mathbf{p}_{\mathbf{2}}^{\prime}$.

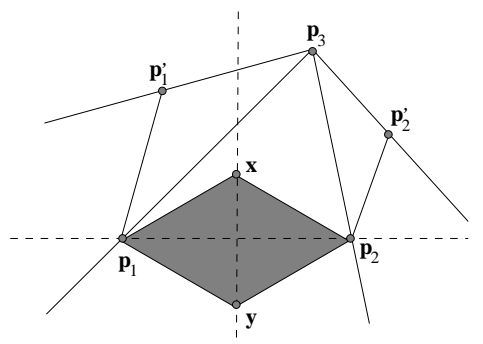

(a)

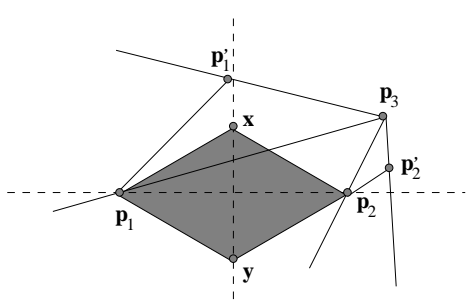

(b)

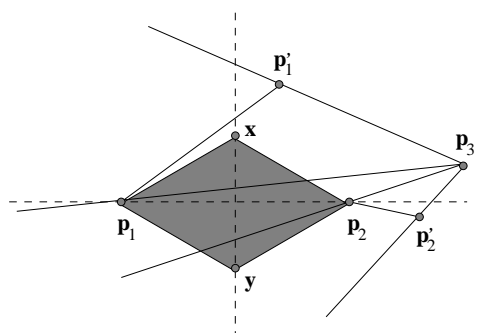

(c)

Fig. 7. The three cases for Lemma 9

Proof: We assume by symmetry that $\mathbf{p}_{\mathbf{3}}$ is to the left of the line $\mathbf{p}_{\mathbf{1}} \mathbf{p}_{\mathbf{2}}$ and to the right of the perpendicular bisector of $\mathbf{p}_{\mathbf{1}} \mathbf{p}_{\mathbf{2}}$. Then $\left\|\mathbf{p}_{\mathbf{1}} \mathbf{p}_{\mathbf{3}}\right\| \geq\left\|\mathbf{p}_{\mathbf{2}} \mathbf{p}_{\mathbf{3}}\right\|$. Since $\mathbf{p}_{\mathbf{3}}$ is outside $L\left(\mathbf{p}_{\mathbf{1}} \mathbf{p}_{\mathbf{2}}\right),\left\|\mathbf{p}_{\mathbf{1}} \mathbf{p}_{\mathbf{3}}\right\| \geq\left\|\mathbf{p}_{\mathbf{1}} \mathbf{p}_{\mathbf{2}}\right\|$ and $\angle \mathbf{p}_{1} \mathbf{p}_{\mathbf{3}} \mathbf{p}_{\mathbf{2}}<\frac{\pi}{2}$. 
Therefore,

$$
\begin{aligned}
& \angle \mathbf{p}_{\mathbf{1}}^{\prime} \mathbf{p}_{\mathbf{3}} \mathbf{p}_{\mathbf{2}}^{\prime}<\frac{\pi}{2}+\frac{\pi}{6}+\frac{\pi}{6}=\frac{5 \pi}{6}, \\
& \angle \mathbf{p}_{\mathbf{1}} \mathbf{p}_{\mathbf{2}} \mathbf{p}_{\mathbf{3}} \geq \frac{\pi}{3} \\
& \angle \mathbf{p}_{\mathbf{2}} \mathbf{p}_{\mathbf{1}} \mathbf{p}_{\mathbf{3}}<\frac{\pi}{2}
\end{aligned}
$$

Let $\mathbf{x}$ and $\mathbf{y}$ be the other two vertices of $D\left(\mathbf{p}_{\mathbf{1}} \mathbf{p}_{\mathbf{2}}\right)$ which lie between the left side and right side respectively of the line $\mathbf{p}_{\mathbf{1}} \mathbf{p}_{\mathbf{2}}$. It's sufficient to show that both $\mathbf{x}$ and $\mathbf{y}$ are within $\measuredangle \mathbf{p}_{\mathbf{1}}^{\prime} \mathbf{p}_{\mathbf{3}} \mathbf{p}_{\mathbf{2}}^{\prime}$. This is true when $\angle \mathbf{p}_{\mathbf{2}} \mathbf{p}_{\mathbf{1}} \mathbf{p}_{\mathbf{3}} \geq \frac{\pi}{\mathbf{6}}$ (see Figure $7\left(\right.$ a)). So we assume that $\angle \mathbf{p}_{2} \mathbf{p}_{\mathbf{1}} \mathbf{p}_{\mathbf{3}}<\frac{\pi}{6}$. In this case $\mathbf{x}$ is within $\triangle \mathbf{p}_{\mathbf{1}} \mathbf{p}_{\mathbf{3}} \mathbf{p}_{\mathbf{1}}^{\prime}$, and thus within $\measuredangle \mathbf{p}_{\mathbf{1}}^{\prime} \mathbf{p}_{\mathbf{3}} \mathbf{p}_{\mathbf{2}}^{\prime}$, from Lemma 8 and $\left\|\mathbf{p}_{\mathbf{1}} \mathbf{p}_{\mathbf{3}}\right\| \geq\left\|\mathbf{p}_{\mathbf{1}} \mathbf{p}_{\mathbf{2}}\right\|$. If $\angle \mathbf{p}_{\mathbf{1}} \mathbf{p}_{\mathbf{2}} \mathbf{p}_{\mathbf{3}} \leq \frac{\mathbf{5 \pi}}{\mathbf{6}}$, then $\mathbf{y}$ is within $\measuredangle \mathbf{p}_{\mathbf{1}}^{\prime} \mathbf{p}_{\mathbf{3}} \mathbf{p}_{\mathbf{2}} \subseteq \measuredangle \mathbf{p}_{\mathbf{1}}^{\prime} \mathbf{p}_{\mathbf{3}} \mathbf{p}_{\mathbf{2}}^{\prime}$ (see Figure $7(\mathrm{~b})$ ). If $\angle \mathbf{p}_{1} \mathbf{p}_{2} \mathbf{p}_{3}>\frac{5 \pi}{6}$, then

$$
\begin{aligned}
\angle \mathbf{p}_{3} \mathbf{p}_{2} \mathbf{y} & =\mathbf{2} \pi-\angle \mathbf{p}_{1} \mathbf{p}_{2} \mathbf{p}_{3}-\angle \mathbf{p}_{1} \mathbf{p}_{2} \mathbf{y} \\
& \geq \mathbf{2} \pi-\pi-\frac{\pi}{\mathbf{6}}=\frac{\mathbf{5} \pi}{\mathbf{6}}
\end{aligned}
$$

which implies that the ray $\mathbf{p}_{\mathbf{2}} \mathbf{y}$ does not intersect with the ray $\mathbf{p}_{\mathbf{3}} \mathbf{p}_{\mathbf{2}}^{\prime}$ (see Figure $7(\mathrm{c})$ ). So $\mathbf{y}$ is within $\measuredangle \mathbf{p}_{\mathbf{1}}^{\prime} \mathbf{p}_{\mathbf{3}} \mathbf{p}_{\mathbf{2}}^{\prime}$. Therefore, in either case both $\mathbf{x}$ and $\mathbf{y}$ are within $\measuredangle \mathbf{p}_{\mathbf{1}}^{\prime} \mathbf{p}_{\mathbf{3}} \mathbf{p}_{\mathbf{2}}^{\prime}$. This completes the proof.

Now we are ready to prove the "disjoint diamond" property of Euclidean MSTs.

Lemma 10: In any Euclidean MST, the two diamonds determined by any two edges are disjoint.

Proof: The lemma is true when two edges are incident to a common vertex as the angle between them is at least $\frac{\pi}{3}$. So we consider two edges $\mathbf{p}_{\mathbf{1}} \mathbf{p}_{\mathbf{2}}$ and $\mathbf{q}_{\mathbf{1}} \mathbf{q}_{\mathbf{2}}$ with distinct endpoints. We consider two cases.

Case 1: At least one of $\mathbf{p}_{\mathbf{1}} \mathbf{p}_{\mathbf{2}}$ and $\mathbf{q}_{1} \mathbf{q}_{2}$ does not cross the perpendicular bisector of the other. Without loss of generality, assume that $\mathbf{q}_{\mathbf{1}}$ and $\mathbf{q}_{\mathbf{2}}$ lie in the same side of the perpendicular bisector of $\mathbf{p}_{\mathbf{1}} \mathbf{p}_{\mathbf{2}}$ as $\mathbf{p}_{\mathbf{1}}$ (see Figure $8(\mathrm{a})$ ). Let $\mathbf{q}_{\mathbf{1}}^{\prime}$ ( $\mathbf{q}_{\mathbf{2}}^{\prime}$ respectively) be the vertex of $\mathbf{D}\left(\mathbf{p}_{\mathbf{1}} \mathbf{q}_{\mathbf{1}}\right)$ $\left(\mathbf{D}\left(\mathbf{p}_{\mathbf{1}} \mathbf{q}_{\mathbf{2}}\right)\right.$ respectively) which is lies on the opposite side of the line $\mathbf{p}_{\mathbf{1}} \mathbf{q}_{\mathbf{1}}\left(\mathbf{p}_{\mathbf{1}} \mathbf{q}_{\mathbf{2}}\right.$ respectively) from $\mathbf{q}_{\mathbf{2}}$ ( $\mathbf{q}_{\mathbf{1}}$ respectively). Then from Lemma $9, \mathbf{D}\left(\mathbf{q}_{1} \mathbf{q}_{2}\right) \subseteq \measuredangle \mathbf{q}_{1}^{\prime} \mathbf{p}_{2} \mathbf{q}_{2}^{\prime}$. On the other hand, since both $\mathbf{q}_{1}$ and $\mathbf{q}_{2}$ are outside $\mathbf{L}\left(\mathbf{p}_{\mathbf{1}} \mathbf{p}_{\mathbf{2}}\right), \mathbf{D}\left(\mathbf{p}_{\mathbf{1}} \mathbf{p}_{\mathbf{2}}\right)$ is outside $\measuredangle \mathbf{q}_{\mathbf{1}}^{\prime} \mathbf{p}_{\mathbf{2}} \mathbf{q}_{\mathbf{2}}^{\prime}$. Thus $\mathbf{D}\left(\mathbf{p}_{\mathbf{1}} \mathbf{p}_{\mathbf{2}}\right)$ and $\mathbf{D}\left(\mathbf{q}_{1} \mathbf{q}_{2}\right)$ are disjoint.

Case 2: Both $\mathbf{p}_{\mathbf{1}} \mathbf{p}_{\mathbf{2}}$ and $\mathbf{q}_{\mathbf{1}} \mathbf{q}_{\mathbf{2}}$ cross the perpendicular bisector of the other. Without loss of generality, assume that $\mathbf{q}_{1}$ lies in the same side of the perpendicular bisector of $\mathbf{p}_{\mathbf{1}} \mathbf{p}_{\mathbf{2}}$ as $\mathbf{p}_{\mathbf{1}}$ (see Figure 8(b)). Then $\mathbf{p}_{\mathbf{1}}$ must lie in the same side of the perpendicular bisector of $\mathbf{q}_{\mathbf{1}} \mathbf{q}_{\mathbf{2}}$ as $\mathbf{q}_{\mathbf{1}}$, for otherwise

$$
\left\|\mathbf{p}_{\mathbf{2}} \mathbf{q}_{\mathbf{1}}\right\|>\left\|\mathbf{p}_{\mathbf{1}} \mathbf{q}_{\mathbf{1}}\right\|>\left\|\mathbf{p}_{\mathbf{1}} \mathbf{q}_{\mathbf{2}}\right\|>\left\|\mathbf{p}_{\mathbf{2}} \mathbf{q}_{\mathbf{2}}\right\|,
$$

i.e., both $\mathbf{p}_{\mathbf{1}}$ and $\mathbf{p}_{\mathbf{2}}$ lie in the same side of the perpendicular bisector of $\mathbf{q}_{1} \mathbf{q}_{2}$ as $\mathbf{q}_{2}$, which contradicts to the assumption. Since $\mathbf{q}_{2}$ is outside $\mathbf{L}\left(\mathbf{p}_{\mathbf{1}} \mathbf{p}_{\mathbf{2}}\right)$ and

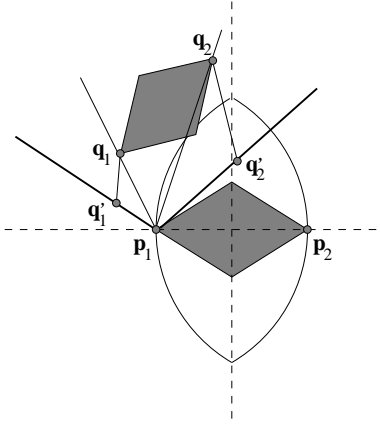

(a)

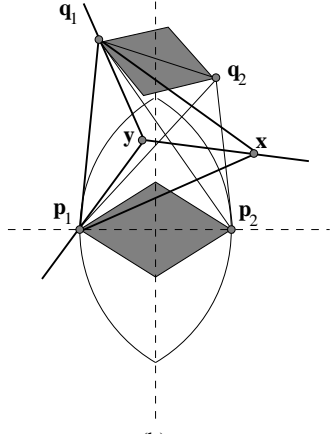

(b)
Fig. 8. Two cases for Lemma 10.

$\left\|\mathbf{p}_{1} \mathbf{q}_{2}\right\|>\left\|\mathbf{p}_{\mathbf{2}} \mathbf{q}_{2}\right\|$, we have $\left\|\mathbf{p}_{1} \mathbf{q}_{2}\right\|>\left\|\mathbf{p}_{1} \mathbf{p}_{\mathbf{2}}\right\|$. As $\left\|\mathbf{p}_{1} \mathbf{q}_{2}\right\|>\left\|\mathbf{p}_{1} \mathbf{q}_{1}\right\|, \mathbf{q}_{2}$ is outside $\triangle \mathbf{q}_{1} \mathbf{p}_{1} \mathbf{p}_{\mathbf{2}}$. Similarly, any of these four points $\mathbf{p}_{1}, \mathbf{p}_{\mathbf{2}}, \mathbf{q}_{\mathbf{1}}$ and $\mathbf{q}_{\mathbf{2}}$ is outside the triangle determined by the other three points. This implies that the convex hull determined by these four points is a quadrilateral. Note that $\mathbf{p}_{\mathbf{1}} \mathbf{p}_{\mathbf{2}}$ and $\mathbf{q}_{\mathbf{1}} \mathbf{q}_{\mathbf{2}}$ can't be the two diagonals of the quadrilateral as they do not cross each other. Neither can be $\mathbf{p}_{\mathbf{1}} \mathbf{q}_{\mathbf{1}}$ and $\mathbf{p}_{\mathbf{2}} \mathbf{q}_{\mathbf{2}}$ as they are separated by the perpendicular bisector of $\mathbf{p}_{\mathbf{1}} \mathbf{p}_{\mathbf{2}}$. Thus the two diagonals must be $\mathbf{p}_{\mathbf{1}} \mathbf{q}_{\mathbf{2}}$ and $\mathbf{p}_{\mathbf{2}} \mathbf{q}_{1}$, and consequently the boundary of the quadrilateral is $\mathbf{p}_{\mathbf{1}} \mathbf{p}_{\mathbf{2}} \mathbf{q}_{\mathbf{2}} \mathbf{q}_{\mathbf{1}}$. From the previous argument its four sides are all less than its two diagonals, and hence its four inner angles are all more than $\frac{\pi}{3}$. Without loss of generality, we assume that $\left\|\mathbf{p}_{\mathbf{1}} \mathbf{q}_{\mathbf{1}}\right\| \geq\left\|\mathbf{p}_{\mathbf{2}} \mathbf{q}_{\mathbf{2}}\right\|$. Then $\left\|\mathbf{p}_{\mathbf{1}} \mathbf{q}_{\mathbf{1}}\right\| \geq\left\|\mathbf{p}_{\mathbf{1}} \mathbf{p}_{\mathbf{2}}\right\|$, for otherwise $\mathbf{q}_{\mathbf{1}}$ would be inside $\mathbf{B}\left(\mathbf{p}_{\mathbf{1}},\left\|\mathbf{p}_{\mathbf{1}} \mathbf{p}_{\mathbf{2}}\right\|\right)$ and $\mathbf{q}_{2}$ would be inside $\mathbf{B}\left(\mathbf{p}_{\mathbf{2}},\left\|\mathbf{p}_{\mathbf{1}} \mathbf{p}_{\mathbf{2}}\right\|\right)$, which is impossible. Similarly, $\left\|\mathbf{p}_{\mathbf{1}} \mathbf{q}_{\mathbf{1}}\right\| \geq\left\|\mathbf{q}_{\mathbf{1}} \mathbf{q}_{\mathbf{2}}\right\|$. Therefore, both $\angle \mathbf{q}_{\mathbf{1}} \mathbf{p}_{\mathbf{1}} \mathbf{q}_{\mathbf{2}}$ and $\angle \mathbf{p}_{1} \mathbf{q}_{1} \mathbf{p}_{\mathbf{2}}$ are less than $\frac{\pi}{3}$. Since both $\angle \mathbf{q}_{2} \mathbf{p}_{\mathbf{1}} \mathbf{p}_{\mathbf{2}}$ and $\angle \mathbf{p}_{2} \mathbf{q}_{1} \mathbf{q}_{2}$ are less than $\frac{\pi}{2}$, we have

$$
\angle \mathbf{q}_{1} \mathbf{p}_{1} \mathbf{p}_{2}, \angle \mathbf{p}_{1} \mathbf{q}_{1} \mathbf{q}_{2} \in\left(\frac{\pi}{3}, \frac{5 \pi}{6}\right) .
$$

Let $\mathbf{x}$ be the point inside $\measuredangle \mathbf{q}_{\mathbf{1}} \mathbf{p}_{\mathbf{1}} \mathbf{p}_{\mathbf{2}}$ such that $\triangle \mathbf{p}_{\mathbf{1}} \mathbf{q}_{\mathbf{1}} \mathbf{x}$ is equilateral. Then both $\mathbf{p}_{\mathbf{1}} \mathbf{p}_{\mathbf{2}}$ and $\mathbf{q}_{\mathbf{1}} \mathbf{q}_{\mathbf{2}}$ are outside $\triangle \mathbf{p}_{1} \mathbf{q}_{1} \mathbf{x}$. In addition,

$$
\angle \mathrm{xp}_{1} \mathbf{p}_{2}, \angle \mathrm{xq}_{1} \mathbf{q}_{2} \in\left(\mathbf{0}, \frac{\pi}{2}\right)
$$

and

$$
\left\|\mathbf{p}_{1} \mathbf{x}\right\| \geq\left\|\mathbf{p}_{\mathbf{1}} \mathbf{p}_{\mathbf{2}}\right\|,\left\|\mathbf{q}_{1} \mathbf{x}\right\| \geq\left\|\mathbf{q}_{1} \mathbf{q}_{\mathbf{2}}\right\| .
$$

Let $\mathbf{y}$ be the center of $\triangle \mathbf{p}_{\mathbf{1}} \mathbf{q}_{\mathbf{1}} \mathbf{x}$. Then from Lemma 8 ,

$$
\mathbf{D}\left(\mathbf{p}_{1} \mathbf{p}_{2}\right) \subseteq \measuredangle \mathbf{p}_{1} \mathbf{y x}, \mathbf{D}\left(\mathbf{q}_{1} \mathbf{q}_{2}\right) \subseteq \measuredangle \mathbf{q}_{1} \mathbf{y x} .
$$

This implies that $\mathbf{D}\left(\mathbf{p}_{\mathbf{1}} \mathbf{p}_{\mathbf{2}}\right)$ and $\mathbf{D}\left(\mathbf{q}_{\mathbf{1}} \mathbf{q}_{\mathbf{2}}\right)$ are disjoint.

Let $P$ be any point set of radius one. According to Lemma 10, the total area of the diamonds through the 
edges in $m s t(P)$ equals to

$$
\frac{\sqrt{3}}{6} \sum_{e \in m s t(P)}\|e\|^{2} .
$$

Let $\mathbf{p}$ be the point in $P$ such that all points in $P$ have distance of at most one from $\mathbf{p}$. Since all edges have lengths of at most one, all diamonds are contained in $\mathbf{B}\left(\mathbf{p}, \frac{\mathbf{2}}{\sqrt{3}}\right)$. This implies that

$$
\frac{\sqrt{3}}{6} \sum_{e \in m s t(P)}\|e\|^{2} \leq \pi\left(\frac{2}{\sqrt{3}}\right)^{2}=\frac{4 \pi}{3} .
$$

Therefore

$$
\sum_{e \in m s t(P)}\|e\|^{2} \leq \frac{8 \pi}{\sqrt{3}} \approx 14.51
$$

This estimation is quite loose and fails in getting the desired 12 upper bound. In the next, we will provide a tighter estimation which can lead to the 12 upper bound.

We observe that the total area of the diamonds is no more than the area of the disk $\mathbf{B}(\mathbf{p}, \mathbf{1})$ plus the sticking-out areas of these diamonds beyond $\mathbf{B}(\mathbf{p}, \mathbf{1})$. Let $\mathbf{D}\left(\mathbf{p}_{\mathbf{1}} \mathbf{p}_{\mathbf{2}}\right)$ be any diamond which sticks out $\mathbf{B}(\mathbf{p}, \mathbf{1})$, and let $\mathbf{q}$ be its vertex which is outside $\mathbf{B}(\mathbf{p}, \mathbf{1})$ (see Figure 9 ). Let $\mathbf{p}_{\mathbf{1}}^{\prime}\left(\mathbf{p}_{\mathbf{2}}^{\prime}\right.$ respectively) be the intersection between $\mathbf{p}_{\mathbf{1}} \mathbf{q}$ $\left(\mathbf{p}_{2} \mathbf{q}\right.$ respectively) and the boundary of $\mathbf{B}(\mathbf{p}, \mathbf{1})$. Then the sticking-out area of $\mathbf{D}\left(\mathbf{p}_{\mathbf{1}} \mathbf{p}_{\mathbf{2}}\right)$ can be calculated by subtracting the area of the sector substended by $\mathbf{p} \mathbf{p}_{\mathbf{1}}^{\prime}$ and $\mathbf{p} \mathbf{p}_{2}^{\prime}$ from the area of the quadrilateral $\mathbf{p} \mathbf{p}_{\mathbf{1}}^{\prime} \mathbf{q} \mathbf{p}_{2}^{\prime}$. The area of the quadrilateral $\mathbf{p p}_{\mathbf{1}}^{\prime} \mathbf{q p}_{\mathbf{2}}^{\prime}$ can be further calculated by summing up the areas of $\triangle \mathbf{p} \mathbf{p}_{\mathbf{1}}^{\prime} \mathbf{p}_{\mathbf{2}}^{\prime}$ and $\triangle \mathbf{q} \mathbf{p}_{\mathbf{1}}^{\prime} \mathbf{p}_{\mathbf{2}}^{\prime}$. As $\angle \mathbf{p}_{\mathbf{1}}^{\prime} \mathbf{q} \mathbf{p}_{\mathbf{2}}^{\prime}$ is a constant $\frac{2 \pi}{3}$, the area of $\triangle \mathbf{q p}_{\mathbf{1}}^{\prime} \mathbf{p}_{\mathbf{2}}^{\prime}$ is maximized when $\left\|\mathbf{q} \mathbf{p}_{\mathbf{1}}^{\prime}\right\| \geq\left\|\mathbf{q} \mathbf{p}_{\mathbf{2}}^{\prime}\right\|$. Let $\angle \mathbf{p}_{\mathbf{1}}^{\prime} \mathbf{p} \mathbf{p}_{\mathbf{2}}^{\prime}=\alpha$, then $\alpha \in\left(0, \frac{\pi}{3}\right]$ and the sticking-out area of $\mathbf{D}\left(\mathbf{p}_{\mathbf{1}} \mathbf{p}_{\mathbf{2}}\right)$ is a at most

$$
S(\alpha)=\frac{1}{2} \sin \alpha+\frac{\sqrt{3}}{6}(1-\cos \alpha)-\frac{\alpha}{2} .
$$

The area function $S(\alpha)$ has the following nice property.

Lemma 11: For any $\alpha, \beta \in\left(0, \frac{\pi}{3}\right)$,

1. if $\alpha+\beta \leq \frac{\pi}{3}, S(\alpha)+S(\beta) \leq S(\alpha+\beta)$;

2. if $\alpha+\beta \geq \frac{\pi}{3}, S(\alpha)+S(\beta) \leq S\left(\alpha+\beta-\frac{\pi}{3}\right)+S\left(\frac{\pi}{3}\right)$.

Proof: The lemma follows from the following two equality: for any $\alpha$ and $\beta$,

$$
\begin{aligned}
& S(\alpha+\beta)-S(\alpha)-S(\beta) \\
& =\frac{4 \sqrt{3}}{3} \sin \frac{\alpha}{2} \sin \frac{\beta}{2} \sin \left(\frac{\pi}{6}-\frac{\alpha+\beta}{2}\right), \\
& S\left(\alpha+\beta-\frac{\pi}{3}\right)+S\left(\frac{\pi}{3}\right)-S(\alpha)-S(\beta) \\
& =\frac{4 \sqrt{3}}{3} \sin \left(\frac{\alpha+\beta}{2}-\frac{\pi}{6}\right) \sin \left(\frac{\pi}{6}-\frac{\alpha}{2}\right) \sin \left(\frac{\pi}{6}-\frac{\beta}{2}\right) .
\end{aligned}
$$

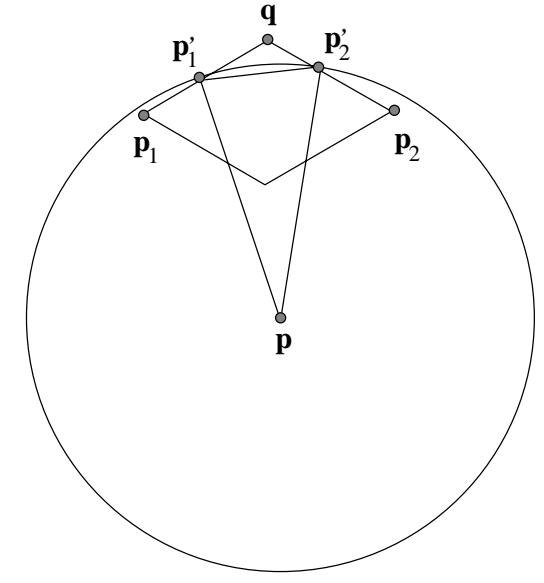

Fig. 9. The calculation of the sticking-out area.

We first prove the first equality.

$$
\begin{aligned}
& S(\alpha+\beta)-S(\alpha)-S(\beta) \\
& =\frac{1}{2}(\sin (\alpha+\beta)-\sin \alpha-\sin \beta)+ \\
& \frac{\sqrt{3}}{6}((\cos \alpha+\cos \beta)-(\cos (\alpha+\beta)+1)) \\
& =\left(\sin \frac{\alpha+\beta}{2} \cos \frac{\alpha+\beta}{2}-\sin \frac{\alpha+\beta}{2} \cos \frac{\alpha-\beta}{2}\right)+ \\
& \frac{\sqrt{3}}{3}\left(\cos \frac{\alpha+\beta}{2} \cos \frac{\alpha-\beta}{2}-\cos ^{2} \frac{\alpha+\beta}{2}\right) \\
& =\sin \frac{\alpha+\beta}{2}\left(\cos \frac{\alpha+\beta}{2}-\cos \frac{\alpha-\beta}{2}\right)+ \\
& \frac{\sqrt{3}}{3} \cos \frac{\alpha+\beta}{2}\left(\cos \frac{\alpha-\beta}{2}-\cos \frac{\alpha+\beta}{2}\right) \\
& =\frac{2 \sqrt{3}}{3} \sin \frac{\alpha}{2} \sin \frac{\beta}{2}\left(\cos \frac{\alpha+\beta}{2}-\sqrt{3} \sin \frac{\alpha+\beta}{2}\right) \\
& =\frac{4 \sqrt{3}}{3} \sin \frac{\alpha}{2} \sin \frac{\beta}{2} \sin \left(\frac{\pi}{6}-\frac{\alpha+\beta}{2}\right)
\end{aligned}
$$

Now we prove the second equality.

$$
\begin{aligned}
& S\left(\alpha+\beta-\frac{\pi}{3}\right)+S\left(\frac{\pi}{3}\right)-S(\alpha)-S(\beta) \\
& =(S(\alpha+\beta)-S(\alpha)-S(\beta))- \\
& \left(S(\alpha+\beta)-S\left(\alpha+\beta-\frac{\pi}{3}\right)-S\left(\frac{\pi}{3}\right)\right) \\
& =\frac{4 \sqrt{3}}{3} \sin \frac{\alpha}{2} \sin \frac{\beta}{2} \sin \left(\frac{\pi}{6}-\frac{\alpha+\beta}{2}\right)- \\
& \frac{4 \sqrt{3}}{3} \sin \left(\frac{\alpha+\beta}{2}-\frac{\pi}{6}\right) \sin \frac{\pi}{6} \sin \left(\frac{\pi}{6}-\frac{\alpha+\beta}{2}\right) \\
& =\frac{4 \sqrt{3}}{3} \sin \left(\frac{\alpha+\beta}{2}-\frac{\pi}{6}\right)\left(\sin \frac{\pi}{6} \sin \left(\frac{\alpha+\beta}{2}-\frac{\pi}{6}\right)-\sin \frac{\alpha}{2} \sin \frac{\beta}{2}\right) \\
& =\frac{2 \sqrt{3}}{3} \sin \left(\frac{\alpha+\beta}{2}-\frac{\pi}{6}\right)\left(\cos \left(\frac{\pi}{3}-\frac{\alpha+\beta}{2}\right)-\cos \frac{\alpha-\beta}{2}\right) \\
& =\frac{4 \sqrt{3}}{3} \sin \left(\frac{\alpha+\beta}{2}-\frac{\pi}{6}\right) \sin \left(\frac{\pi}{6}-\frac{\alpha}{2}\right) \sin \left(\frac{\pi}{6}-\frac{\beta}{2}\right) .
\end{aligned}
$$


Suppose that there are $k$ diamonds which stick out $\mathbf{B}(\mathbf{p}, \mathbf{1})$. For any $1 \leq i \leq k$, let $\alpha_{i}$ be the inner angle of the arc between the two intersection points of the boundary $\mathbf{B}(\mathbf{p}, \mathbf{1})$ and the boundary of the $i$-th stickingout diamond. Then $\alpha_{i} \in\left(0, \frac{\pi}{3}\right]$ and

$$
\sum_{i=1}^{k} \alpha_{i}<2 \pi \text {. }
$$

By repeatedly applying the two inequalities in Lemma 11, the total sticking-out area of the diamonds is

$$
\begin{aligned}
\sum_{i=1}^{k} S\left(\alpha_{i}\right) & \leq\left\lceil\frac{\sum_{i=1}^{k} \alpha_{i}}{\frac{\pi}{3}}\right\rceil S\left(\frac{\pi}{3}\right) \\
& \leq 6 S\left(\frac{\pi}{3}\right)=2 \sqrt{3}-\pi .
\end{aligned}
$$

Thus the total area of diamonds is at most

$$
\pi+2 \sqrt{3}-\pi=2 \sqrt{3} .
$$

Therefore,

$$
\sum_{e \in m s t(P)}\|e\|^{2} \leq \frac{2 \sqrt{3}}{\frac{\sqrt{3}}{6}}=12 .
$$

This completes the proof Theorem 3 .

\section{Summary and Future Works}

In this paper, we have provided theoretical performance analysis the heuristics presented in [8]. The approximation ratio of SPT is at least $\frac{n}{2}$, and thus less favorable from the theoretical perspective. The other two heuristics, MST and BIP, have constant approximation ratios. Specifically, the approximation ratio of MST is between 6 and $c$, which is at most 12; the approximation ratio of the BIP heuristic is between $\frac{13}{3}$ and $c \leq 12$. However, there are still several challenging issues for future research.

First of all, the computational complexity of the Minimum-Energy Broadcasting remains unknown. As mentioned in Section I, the graph-version of this problem is at least as hard as the Connected Dominate Set problem. However, due to its geometric nature, this intractness of the graph version does not imply the same intractness of geometric version which is studied in this paper. Indeed, while Connected Dominating Set problem does not allow a constant-approximation ratio, the geometric version does on the contrary, for example, by MST or BIP.

Secondly, the exact value of the constant $c$ remains unsolved. A tighter upper bound on $c$ can lead to tighter upper bounds on the approximation ratios of both MST and BIP. From the deriving of the 12 upper bound, we observe there are still rooms to improve the upper bound.
For example, it's very unlikely for the diamonds to fill the unit disk fully. At least this is true for small number of nodes. However, the treatment of large number of nodes is quite challenging, and more geometric properties of the Euclidean MSTs have to be explored.

The third interesting problem is how to construct "worse" instances that can lead to better lower bounds on the approximation ratios of both the MST and BIP.

A major challenge, and a topic of continued research, is the development of distributed algorithms of MST and BIP. These algorithms should take advantage of the geometric properties for fast implementation. Furthermore, it is important to study the impact of limited bandwidth and transceiver resources, as well as to develop mechanisms to cope with node mobility [8].

\section{REFERENCES}

[1] V. Chvátal, "A Greedy Heuristic for the Set-Covering Problem", Mathematics of Operations Research, Vol. 4, No. 3, pp. 233-235, 1979

[2] T. J. Cormen, C. E. Leiserson, and R. L. Rivest, Introduction to Algorithms, MIT Press and MxGraw-Hill, 1990.

[3] M. R. Garey, and D. S. Johnson, Computers and Intractability: a Guide to the Theory of NP-Completeness, W. H. Freeman and Company, 1979.

[4] S. Guha, and S. Khuller, "Approximation Algorithms for Connected Dominating Sets". Algorithmica 1998, 20:347-387.

[5] F. P. Preparata and M. I. Shamos, Compuatational Geometry: an Introduction, Springer-Verlag, 1985.

[6] T. S. Rappaport, Wireless Communications: Principles and Practices, Prentice Hall, 1996.

[7] S. Singh, C. S. Raghavendra, and J. Stepanek, "Power-Aware Broadcasting in Mobile Ad Hoc Networks", Proceedings of IEEE PIMRC'99, Osaka, Japan, Sep. 1999.

[8] J.E. Wieselthier, G.D. Nguyen, and A. Ephremides, "On the Construction of energy-Efficient Broadcast and Multicast Trees in Wireless Networks". IEEE Infocom'2000. 